Draft Version SePtember 2, 2021

Preprint typeset using $\mathrm{IAT}_{\mathrm{E}} \mathrm{X}$ style emulateapj v. 5/2/11

\title{
SG1120-1202: MASS-QUENCHING AS TRACKED BY UV EMISSION IN THE GROUP ENVIRONMENT AT $\mathrm{z}=0.37$
}

\author{
Jonathan T. Monroe \\ Physics and Astronomy, Texas A\&M University, College Station, TX 77840, USA \\ KIM-VY H. TRAN \\ Physics and Astronomy, Texas A\&M University, College Station, TX 77840, USA \\ AND \\ Anthony H. Gonzalez \\ Astronomy Department, University of Florida, Gainesville, FL 32611, USA \\ Draft version September 2, 2021
}

\begin{abstract}
We use the Hubble Space Telescope to obtain WFC3/F390W imaging of the supergroup SG1120-1202 at $z=0.37$, mapping the UV emission of 138 spectroscopically confirmed members. We measure total (F390W-F814W) colors and visually classify the UV morphology of individual galaxies as "clumpy" or "smooth." Approximately 30\% of the members have pockets of UV emission (clumpy) and we identify for the first time in the group environment galaxies with UV morphologies similar to the jellyfish galaxies observed in massive clusters. We stack the clumpy UV members and measure a shallow internal color gradient, which indicates unobscured star formation is occurring throughout these galaxies. We also stack the four galaxy groups and measure a strong trend of decreasing UV emission with decreasing projected group distance $\left(R_{\text {proj }}\right)$. We find that the strong correlation between decreasing UV emission and increasing stellar mass can fully account for the observed trend in (F390W-F814W)- $\mathrm{R}_{\text {proj }}$, i.e., mass-quenching is the dominant mechanism for extinguishing UV emission in group galaxies. Our extensive multi-wavelength analysis of SG1120-1202 indicates that stellar mass is the primary predictor of UV emission, but that the increasing fraction of massive (red/smooth) galaxies at $R_{\text {proj }} \lesssim 2 R_{200}$ and existence of jellyfish candidates is due to the group environment.

Subject headings: galaxies: clusters: individual (SG1120-1202) - galaxies: evolution - galaxies: starburst - galaxies: morphology
\end{abstract}

\section{INTRODUCTION}

How galaxies evolve as a function of their local environment continues to be a rich topic of exploration for both observational and theoretical studies. The origins of well-established trends such as the increasing fraction of blue/star-forming/disk-dominated galaxies in galaxy clusters at higher redshift (e.g. Butcher \& Oemler, A. 1978; Dressler 1980; Stanford et al. 1998; Cooper et al. 2010) seem to be rooted in environmental processes. However, dissecting the relative importance of physical mechanisms such as ram pressure stripping Gunn \& Gott III 1972: Abadi et al. 1999), galaxy harassment (Moore et al. 1998), tidal interactions (Byrd \& Valtonen 1990), and gas starvation (Larson et al. 1980, Bekki et al. 2002) remains a challenge.

In the hierarchical formation model (Peebles 1970), galaxy clusters are built by merging smaller groups. Observations of nearby groups show they have more in common with galaxy clusters than with the field population, i.e., higher early-type fractions and lower mean starformation rates than the field (Hashimoto et al. 1998. Zabludoff \& Mulchaey 1998; Tran et al. 2001), and observations at $z \gtrsim 0.2$ highlight the importance of the group environment in transforming late to early-type galaxies (Gallazzi et al. 2009, Iovino et al. 2010: Koyama et al. 2010).

jonathan.monroe@tamu.edu
Motivated by the rapid evolution of the spiral population since $z<1$ (Dressler et al. 1997; Kodama et al. 2001; Postman et al. 2005; Wilman et al. 2008), many investigations focus on the transformation of infalling spirals, either via a rapid, triggered burst of star formation or quenching of subsequent star formation. Simulations are sufficiently advanced that new insight can be obtained by, e.g. comparing star-forming regions and gasloss rates to observations (Tonnesen et al. 2007). Also, galaxy mass may have a more dominant role in quenching star formation when clusters are still assembling (Peng et al. 2010, Muzzin et al. 2012), i.e., when galaxies are in a more group-like environment. However, to connect galaxies evolving in groups to more massive clusters requires identifying the progenitors of local clusters, i.e., galaxy groups at higher redshift.

The supergroup SG1120-1202 (hereafter SG1120) at $z=0.37$ provides a unique laboratory for studying galaxies in a group environment before the groups merge to form a more massive galaxy cluster. SG1120 was discovered in the Las Campanas Deep Cluster Survey (Gonzalez et al. 2001) and is composed of four X-ray luminous galaxy groups that will merge to form a cluster comparable in mass to Coma (Gonzalez et al. 2005). Our extensive multi-wavelength observations show that most of SG1120's members have early-type morphologies ( $>60 \%$; Kautsch et al. 2008) and that most of the S0 members formed before the cluster phase (Just et al. 
2011). However, the fraction of members that are bright $24 \mu \mathrm{m}$ sources is nearly as high as in the field $(\sim 30 \%$ Tran et al. 2009). Spectroscopy with integral field units also show that several SG1120 members have outflowing winds that may aid in quenching star formation (Freeland et al. 2011).

In this paper, we focus on current star formation as traced by UV emission using Hubble Space Telescope imaging with WFC3/F390W. By combining with our existing ACS/F814W mosaic, we obtain high-resolution color maps of the supergroup members to pinpoint (dustfree) star-forming regions and measure internal color gradients. We compare the UV maps to stellar masses and projected group distance to quantify the relative importance of mass versus environmental quenching. Throughout the paper, we use $H_{0}=70 \mathrm{~km} \mathrm{~s}^{-1} \mathrm{Mpc}^{-1}, \Omega_{M}=$ 0.3 and $\Omega_{\Lambda}=0.7$. At $\mathrm{z}=0.37$, this corresponds to a scale of $5.12 \mathrm{kpc} \operatorname{arcsec}^{-1}$ and a look-back time of 4 Gyr.

\section{OBSERVATIONS}

Gonzalez et al. (2005) identified a merging system of four X-ray luminous groups with a total combined mass of $5.3 \times 10^{14} M_{\odot}$ (hereafter called SG1120; see Table 1 for SG1120 properties). The individual galaxy groups lie on the local $\sigma-T$ relation, and a virial analysis using their $\mathrm{X}$-ray masses and relative locations indicates that these groups are bound to each other, likely infalling for the first time, and will merge into a single system by $z=0$. A weak-lensing analysis based on HST imaging supports this picture (Smit et al. 2015), and 174 group galaxies have been spectroscopically confirmed using Magellan, MMT, and VLT/VIMOS.

Previous studies comparing SG1120 to clusters have found comparable fractions of early-type members (Kautsch et al.2008) but also enhanced fractions of $24 \mu \mathrm{m}$ sources (Tran et al. 2009). The high fraction of earlytype galaxies combined with enhanced star formation in SG1120 indicates that the transition to the cluster environment is well underway even in the group environment. The combination of cluster and field-like properties make SG1120 a unique laboratory for investigating how environmental processes drive and/or quench star formation.

\subsection{Hubble Space Telescope Imaging}

We employ HST imaging of an $\sim 8^{\prime} \times 12^{\prime}$ mosaic across three filters: F390W (WFC3/UVIS), F606W (ACS/WFC), and F814W (ACS/WFC) for a total of 44 pointings (combined primary and parallels) during cycles 14 (GO 10499) and 19 (GO 12470). The exposure times in F814W and F390W were $2000 \mathrm{~s}$ and $2610 \mathrm{~s}$, respectively. At $z=0.37$, these three filters probe rest-frame UV and optical emission to track both recent star formation and the existing stellar population. Furthermore, the high resolution of HST/WFC3 resolves the internal structure and the fine details of the individual galaxies. We measure galaxy sizes (radii) using F814W and use F390W to identify compact star-forming regions. The $\mathrm{F} 606 \mathrm{~W}$ is only used to generate the color images for a subset of members; the F606W coverage is not as extensive as F390W and F814W (see Fig. 1).

Of the original sample of 174 spectroscopically confirmed members, we exclude 31 because they do not have imaging in either F814W or F390W. Furthermore, eight galaxies fall near boundaries on the CCD and so our measurements are unreliably noisy, leaving a total of 136 confirmed supergroup galaxies for which we can measure galaxy colors and visually classify their F390W emission. For details on the spectroscopy and stellar mass measurements from multi-band ground-based observations, we refer the reader to Tran et al. (2009).

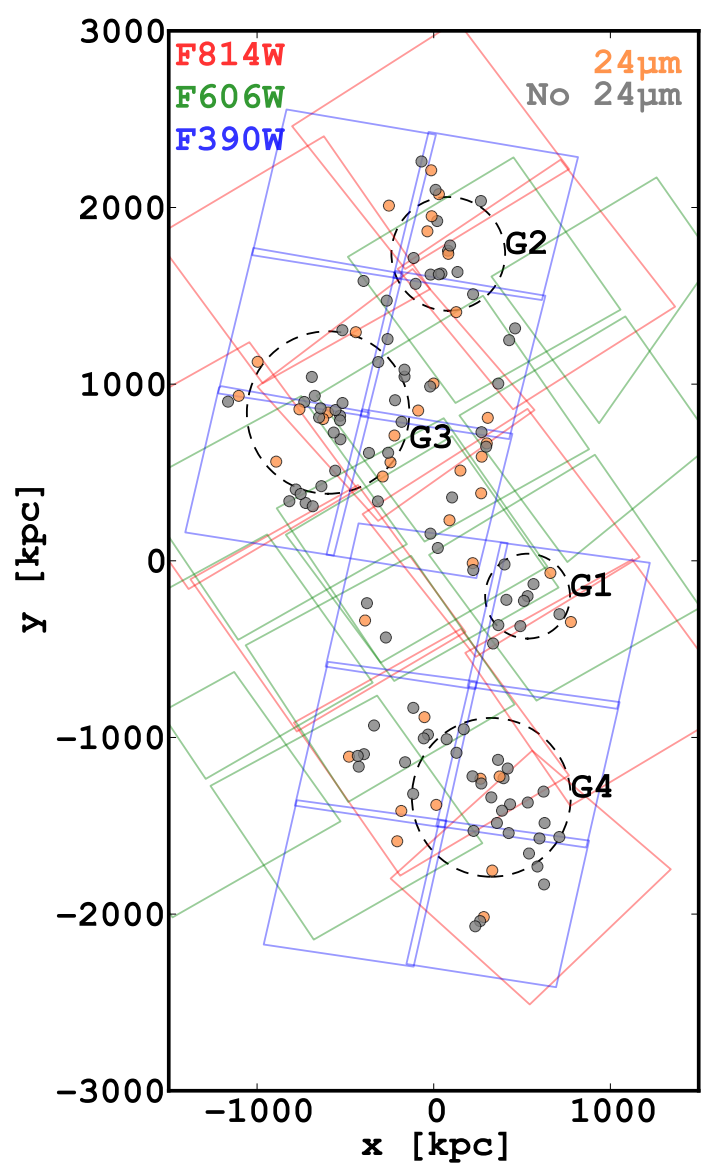

FIG. 1. - Spatial distribution of spectroscopically confirmed members across the entire galaxy supergroup SG1120-1202; north is up and east is to the left. HST coverage in WFC3/F390W, $\mathrm{ACS} / \mathrm{F} 606 \mathrm{~W}$, and $\mathrm{ACS} / \mathrm{F} 814 \mathrm{~W}$ are included in blue, green, and red, respectively. MIPS $24 \mu \mathrm{m}$ detection or non-detection is denoted with orange or gray color, respectively. Black dashed circles of radius $R_{200}$ are centered on each Brightest Group Galaxy (BGG), which are labeled according to the ordering of Tran et al. (2009).

\subsection{Measuring Galaxy Colors}

To measure colors and fluxes via Source Extractor (Bertin \& Arnouts 1996), we first redrizzle our data to align all images. We utilize the default HST pipeline through the calibration and flat-fielding step (flt output images), but redrizzle our data via AstroDrizzle (S. Gonzaga, W. Hack, A. Fruchter 2012) to combine our mosaic with optimized pixel resampling and astrometry. To retain resolution and to ensure detection of small-scale star forming regions, we apply the finest pixel resolution, 0.'0396/pixel (WFC3/UVIS), and resample the other two datasets (ACS F606W and F814W) from their native scale $(0 . \prime 05 \%$ pixel $)$ to match. Resampling all images to a common resolution facilitates consistent color measure- 
TABLE 1

SG1120-1202 Constituent GRoup PROPERTIEs.

\begin{tabular}{lcccccccc} 
Index & $\begin{array}{c}\alpha \\
(\mathrm{J} 2000)\end{array}$ & $\begin{array}{c}\delta \\
(\mathrm{J} 2000)\end{array}$ & $z$ & $\begin{array}{c}T \\
(\mathrm{keV})\end{array}$ & $\begin{array}{c}M \\
\left(M_{\odot}\right)\end{array}$ & $\begin{array}{c}\sigma \\
\left(\mathrm{km} \mathrm{s}^{-1}\right)\end{array}$ & $\begin{array}{c}R_{200} \\
(\mathrm{kpc})\end{array}$ & $\begin{array}{c}N \\
(\text { members })\end{array}$ \\
\hline \hline 1 & $11: 20: 07.48$ & $-12: 05: 09.1$ & 0.3522 & 2.2 & $1.3 \times 10^{14}$ & 303 & 240 & 28 \\
2 & $11: 20: 13.33$ & $-11: 58: 50.6$ & 0.3707 & 1.7 & $8.0 \times 10^{13}$ & 406 & 320 & 27 \\
3 & $11: 20: 22.19$ & $-12: 01: 46.1$ & 0.3712 & 1.8 & $8.9 \times 10^{13}$ & 580 & 460 & 54 \\
4 & $11: 20: 10.14$ & $-12: 08: 51.6$ & 0.3694 & 3.0 & $2.3 \times 10^{14}$ & 576 & 460 & 47 \\
\hline
\end{tabular}

Note. - Properties summarized from Gonzalez et al. (2005) and Tran et al. (2009)

ments across multi-band imaging. This resampling does not alter colors by more than $2 \%$. We use the finalwht-type parameter to ensure our output weight maps are inverse variance maps as per the expected input to SExtractor (see Skelton et al. 2014 for more details).

We measure colors with SExtractor (Bertin \& Arnouts 1996) in dual-image mode, which uses a reference image (here $\mathrm{F} 814 \mathrm{~W}$ ) for detection and then measures fluxes in the target image (F390W or F814W). Only by using the same detection map can we directly compare colors and sizes across the filters. All pixels that are associated with a galaxy in the detection image (segmentation map) are used in calculating its flux in the target image.

We utilize published values for instrument and filterspecific fields such as zeropoints, FWHM, etc., and use default parameters for all fields except minimum detection. We adjust minimum detection area to prevent faulty detections from entering our catalogs, especially in F390W, where object sizes are substantially smaller and the background noisier. Our choice for detection area includes all galaxy members, while filtering most of the false detections such as those along the imaging boundaries and in inter-chip regions. Table 2 includes the magnitudes in F814W and F390W and half-light radius in $\mathrm{F} 814 \mathrm{~W}$ for all spectroscopically confirmed group galaxies.

\subsection{Identifying UV Emission from Star Formation}

We visually inspect the HST imaging to identify SG1120 members that have bright pockets of UV emission indicative of ongoing (unobscured) star formation. The $\mathrm{O}$ and $\mathrm{B}$ type stars illuminating these regions burn for $\sim 30$ Myrs and, while they constitute less than $7 \%$ of stellar mass (Wuyts et al. 2012), these massive stars effectively trace new sites of unobscured star formation. We note that the absence of OB-type stars does not imply that a galaxy is devoid of star formation, e.g. UV light is easily reprocessed to longer wavelengths by dust that is then detected at $24 \mu \mathrm{m}$.

J.M. and K.T. separately examined the HST imaging in the individual filters as well as in the combined RGB images (see Fig. 2, right). Group members are classified as "clumpy" if UV-bright regions exist throughout the galaxy and "smooth" if there are no such regions. Several galaxies are edge-on disks and therefore cannot be reliably classified; these are denoted as "none."

The right side of Figure 2 shows RGB images for a sample of each visual classification, and Table 2 includes the classification for each galaxy, including "jellyfish" candidates. Because of their increased rest-frame UV flux, regions of ongoing (unobscured) star formation are visible as blue pockets of light (F390W) in the RGB images.
Approximately $30 \%$ of the supergroup have "clumpy" UV emission.

We use the F390W imaging to visually identify jellyfish candidates, which are characterized by asymmetric ultraviolet clumps with trails of knots and filaments (Smith et al. 2010). The high resolution of HST imaging allows us to visually identify such features according to the criteria used in the literature (e.g. Smith et al. 2010; Owers et al. 2012; Ebeling et al. 2014) even at $z \sim 0.37$. In Fig. 3 we include two such examples with RGB thumbnails and grayscale images of F814W and F390W. The side-by-side comparison allows the rest-frame UV emission of trails to directly contrast the underlying stellar population.

\subsection{Spitzer/MIPS $24 \mu m$ Fluxes}

We use the Spitzer MIPS $24 \mu \mathrm{m}$ fluxes from Saintonge et al. (2008) and Tran et al. (2009). To summarize, the $24 \mu \mathrm{m}$ observations were retrieved from the Spitzer archive and spectral energy distributions were fit to determine the IR luminosities and corresponding star-formation rates of each source (see Saintonge et al. 2008 for details). At $z \sim 0.37$, the $80 \%$ completeness limit corresponds to $\log (\mathrm{LIR})\left[\mathrm{ergs}^{-1}\right]=43.8$ or, equivalently, a star-formation rate of $3 M_{\odot}$. Figure 1 shows the spatial distribution of the $24 \mu \mathrm{m}$-detected group galaxies that also have the required F390W and F814W imaging. Following Tran et al. (2009), we attribute the IR emission to dusty star formation (contamination by active galactic nuclei is $<3 \%$ ).

\section{RESULTS}

\subsection{UV Morphology Correlates with Projected Distance}

As shown by Kautsch et al. (2008), the trend of increasing early-type fraction in denser environments is already established in the SG1120. We build on this morphology-density relation by examining the spatial distribution of supergroup galaxies with pockets of UV emission ("clumpy"; Fig. 2, right). We stack all four galaxy groups and find that the fraction of members with patches of UV emission (clumpy) increases with increasing distance from the group centers (Figure 4). The tendency for clumpy members to be at larger projected distances from the cores suggests that newly accreted group galaxies with UV-bright star-forming regions are extinguished as they fall into the groups. While there are a handful of members with "clumpy" emission in the group cores, their position may be due primarily to projection effects.

\subsection{Color-Magnitude Diagrams (CMDs)}




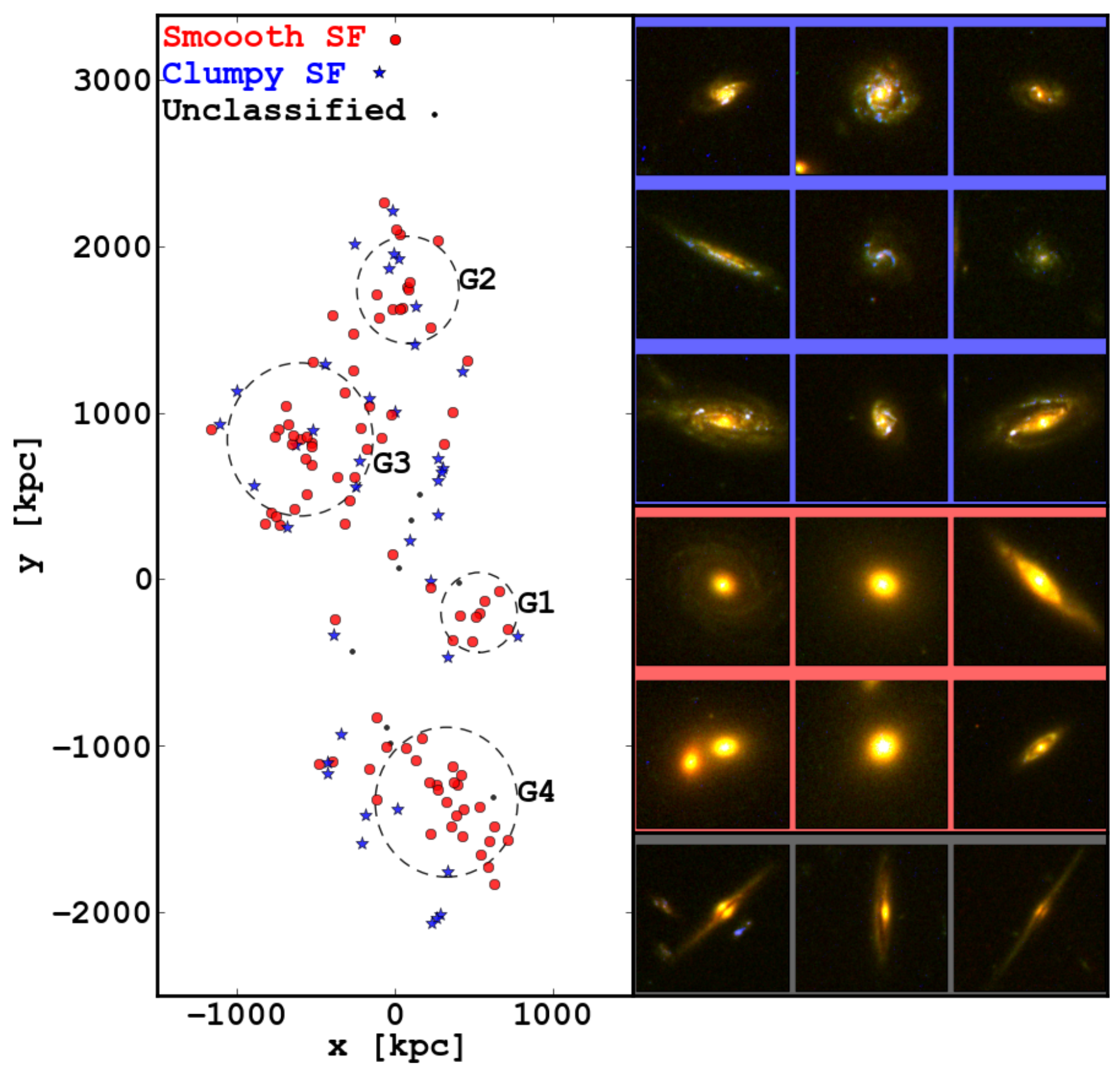

FIG. 2.- Spatial distribution of supergroup galaxies where the members are separated by visual identification of UV emission as traced by F390W. The dashed circles correspond to $R_{200}$ for each group (see Table 1). Galaxies with pockets of UV emission in their disks are considered "clumpy" and those with smooth radial profiles are "smooth". Examples of clumpy and smooth classifications are shown as color images (right subpanels) that are generated by combining HST F814W, F606W, and F390W. Unclassified objects are typically dust-obscured edge-on galaxies such as those in the bottom thumbnails. 

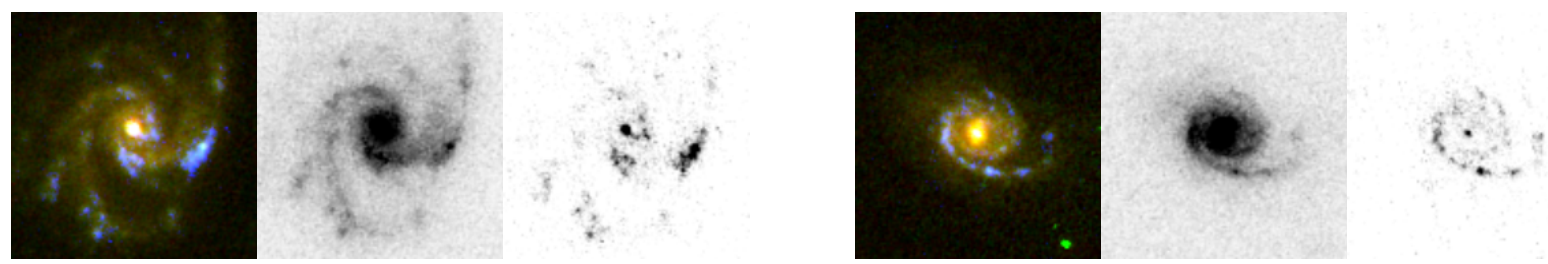

FiG. 3.- Examples of member galaxies (left: J112013.2-120345.2, right:J112014.5-115808.9), which we visually characterize as jellyfish candidates (Smith et al. 2010). Color images (left) are generated by combining HST F814W, F606W, and F390W. Grayscale images of F814W imaging (middle) and F390W imaging (right). The presence of asymmetric clumps and trails of knots and filaments serves as the defining characteristics of such galaxies. Table 2 includes labels denoting all jellyfish candidates.

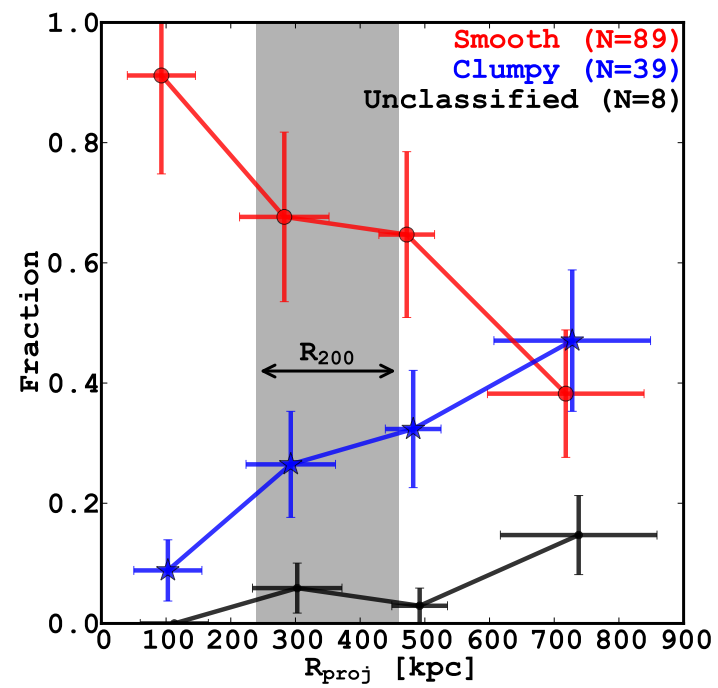

FIG. 4.- We stack the four groups in SG1120 and show the fraction of members with pockets of UV emission (clumpy) relative to those without (smooth) as a function of the projected group-centric distance. The binned points are offset by $10 \mathrm{kpc}$ for clarity, and the $R_{200}$ range for the four groups (Table 1 ) is shown as a vertical band. Clumpy galaxies are characterized by UV emission from ongoing star formation at extended radii and their fraction increases at larger distances from the group core. Unclassified galaxies are mostly comprised of edge-on disks, where star formation is obscured by the disk; their fraction also increases with increasing distance from the core.

CMDs are an efficient method for tracing how a galaxy evolves as its stellar population ages. As a galaxy's starformation rate decreases, its colors redden and move it from the blue cloud to the red sequence within the CMD. Rest-frame unobscured UV emission is particularly effective at measuring unobscured SF rates because it is generated by the youngest and most massive main-sequence OB-type stars with lifetimes of $<30 \mathrm{Myr}$. In Figure[5, we compare the (F390W-F814W) distributions for different populations of supergroup members based on their IR $(24 \mu \mathrm{m})$ and UV (F390W) emission.

In Fig. 5 (top), we compare the color distribution of $24 \mu \mathrm{m}$ members to those that are undetected. Although $24 \mu \mathrm{m}$ and UV flux both trace star formation (Kennicutt Jr et al. 2012), the correlation with (F390W-F814W) colors can be weak because UV emission is easily reprocessed by dust, which then emits in the far-infrared (Meurer et al. 1999). The 28 IR-detected supergroup galaxies above our completeness limit of $m_{F 814 W}>21.2$ are about 0.3 dex bluer than the non-IR members, and a KS test confirms that the difference is significant $(>3 \sigma)$.
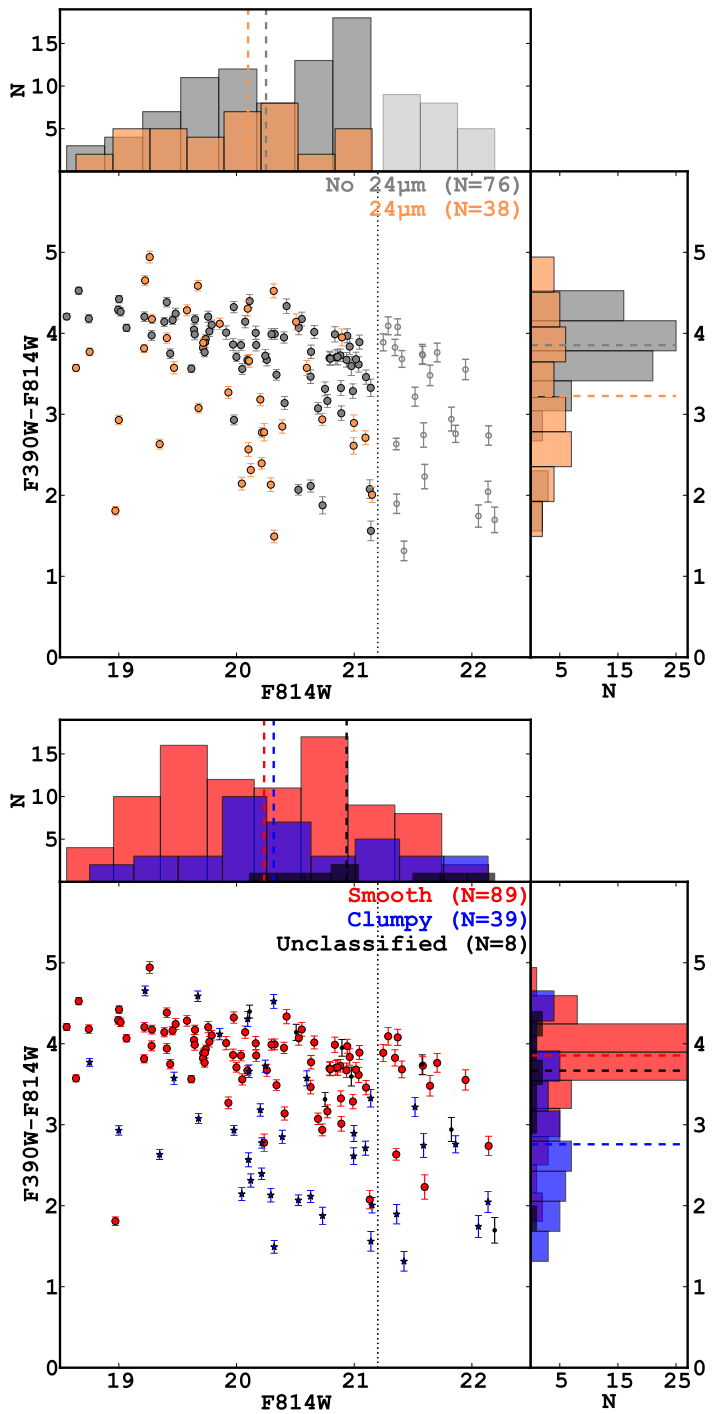

Fig. 5.- Color-magnitude diagram (top) for supergroup galaxies comparing $24 \mu \mathrm{m}$ (orange filled circles) and non-detected (gray filled circles) members; galaxies fainter than the spectroscopic completeness limit of $m_{F 814 W}=21.2$ (dotted vertical line) are shown as open gray circles. Also shown is the CMD (bottom) for members with pockets of UV emission (clumpy; blue stars) or smooth profiles (red filled circles). The upper and right side histograms show the $m_{814}$ and (F390W-F814W) distributions for the different galaxy populations; the vertical dashed lines correspond to the average values. There is a larger difference in average (F390W-F814W) color between clumpy vs. smooth galaxies ( $\sim 1.0$ dex $)$ compared to $24 \mu m$ vs. non- $24 \mu m$ ( $\sim 0.3 \mathrm{dex})$.

Note that while the $24 \mu \mathrm{m}$ members tend to be blue, they 
also span the full range in (F390W-F814W) color and include some of the reddest galaxies.

The transition from star-forming to quiescent galaxy also usually corresponds to changes in morphology from late- to early-type (van Dokkum et al. 1998, Balogh et al. 2004 Bell et al. 2004). We compare the supergroup galaxies based on their clumpy/smooth classification (Figure 5 bottom) and find that these two populations differ in average (F390W-F814W) color by $\sim 1$ dex, i.e. more than three times larger than the IR vs. nonIR members. When considering all of the members, the average (F390W-F814W) colors for smooth and clumpy are $3.76 \pm 0.08$ and $2.79 \pm 0.09$ respectively; if we consider only $m_{F 814 W}<21.2$, the average $(\mathrm{F} 390 \mathrm{~W}-\mathrm{F} 814 \mathrm{~W})$ are $3.81 \pm 0.08$ and $2.92 \pm 0.09$.

To summarize, the supergroup galaxies with smooth light profiles tend to be very uniform in color, to have color shallow gradients, to be red, to have early-type morphologies, and to populate the group cores (Figs. 2 \& 4. Kautsch et al. 2008). In contrast, the clumpy galaxies contain both red and blue components, span the range in galaxy color, tend to have prominant disks, and are mostly outside the group cores.

\subsection{Total Galaxy Color Correlates with Projected Distance}

In Fig. 6, we compare how total (F390W-F814W) color and local environment are correlated by stacking the four groups into a single system and using the projected radius $\left(R_{\text {proj }}\right)$ from the nearest Brightest Group Galaxy (defined as the group center). The projected radius serves as a proxy for environmental density. For an analysis of how the $24 \mu \mathrm{m}$ emission depends on local environment in SG1120, we refer to Tran et al. (2009).

Supergroup galaxies in the group cores are redder than those at larger distances. A least-squares fit to the total galaxy color and projected distance for the stacked groups (Fig. 6. black line) measures a strong correlation of

$$
(\mathrm{F} 390 \mathrm{~W}-\mathrm{F} 814 \mathrm{~W})=8.56 \times 10^{-4}\left(\frac{R_{\text {proj }}}{\mathrm{kpc}}\right)+3.82
$$

at $>3 \sigma$ confidence (errors in slope and offset are 2.64 and 0.12 , respectively). Least-squares fits to the individual groups measure the same general trend. Figure 6 includes the error bars that represent each bin's range in $\mathrm{R}_{\text {proj }}$ and standard deviation in (F390W-F814W). As galaxies approach their group cores, their increasingly red (F390W-F814W) colors indicate that their star formation is quenching.

\subsection{Correlations with Stellar Mass}

To test if the correlation between total galaxy color and projected distance in SG1120 (see 33.3 is driven by an increasing number of massive, passive galaxies in the group cores, we compare (F390W-F814W) color to stellar mass $\left(M_{\star}\right)$ in Fig. 7. A least-squares fit confirms a strong correlation of

$$
(\mathrm{F} 390 \mathrm{~W}-\mathrm{F} 814 \mathrm{~W})=0.95 \times \log \left[M_{\star} / 10^{10} M_{\odot}\right]+3.09
$$

(errors in slope and offset are 0.08 and 0.06 , respectively) shown as a solid line in Fig. 7. more massive

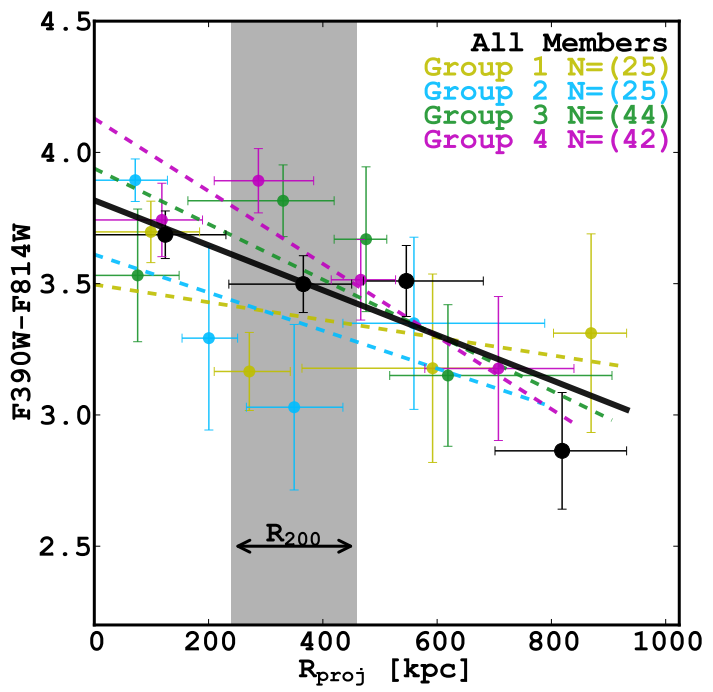

FIG. 6. - Total (F390W-F814W) color for individual supergroup galaxies compared to their projected distance (Rproj) for the group stack; the vertical dashed line corresponds to a projected distance of $400 \mathrm{kpc}$ which is approximately $R_{200}$ (see Table 1 ). We measure a strong correlation ( $>3 \sigma$ confidence) between (F390W-F814W) color and projected distance $\left(\mathrm{R}_{\text {proj }}\right)$ : members are redder in the group cores. The error bars in $\mathrm{R}_{\text {proj }}$ and (F390W-F814W) color represent, respectively, the bin range and standard deviation within the bin.

supergroup members have redder (F390W-F814W) colors. Although 22 galaxies are below our spectroscopic completeness limit (which we take to estimate our mass completeness), the slope of Eq. 2 changes by less than $1 \%$ when we exclude galaxies below this limit.

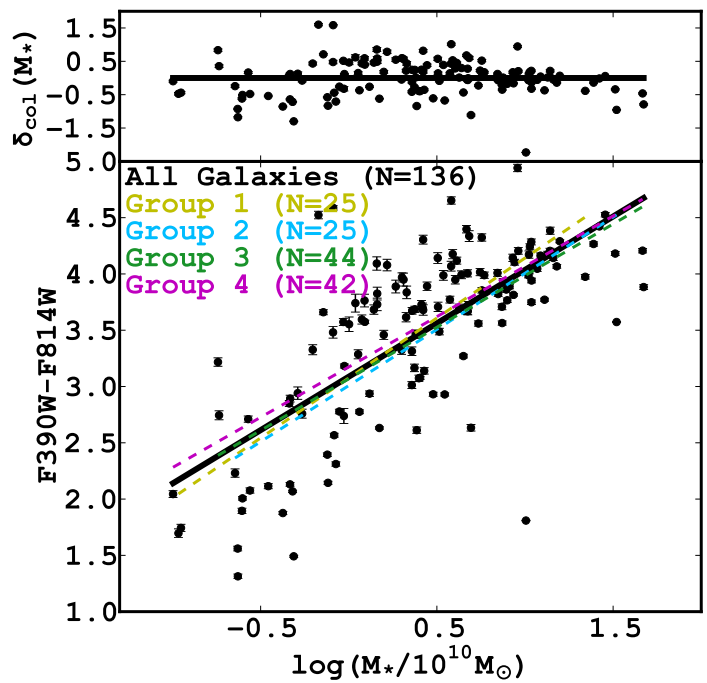

FIG. 7.- In SG1120, the total (F390W-F814W) color for individual members is tightly correlated with their stellar mass in all of the groups (bottom; colored lines are least-squares fits to the separate groups). A least-squares fit to the supergroup stack (black line) has mean residuals within $\sim 0.5$ dex (top). The strong correlation between (F390W-F814W) color and stellar mass is what drives the observed correlation between (F390W-F814W) and projected group distance (Fig. 6): massive supergroup galaxies tend to be red and tend to be in the group cores.

We now combine the measured relation between total galaxy color and stellar mass (Fig. 7) with the measured relation between total galaxy color and projected group 
distance (Fig. 6). Mass and projected group distance correlate with a slope of $-9.02 \pm 1.95 \times 10^{-4}$, but we seek to separate the effects of these properties on total galaxy color. For each supergroup galaxy, we calculate a color deviation $\delta_{\text {col }}\left(M_{\star}\right)$ defined as the difference between the galaxy's measured (F390W-F814W) color and the expected value given its stellar mass from Eq. 2. Once we account for the correlation between total galaxy color and stellar mass, the trend between (F390W-F814W) color and $\mathrm{R}_{\text {proj }}$ disappears (Fig. 8). Least-squares fits to $\delta_{\mathrm{col}}\left(M_{\star}\right)$ and $\mathrm{R}_{\text {proj }}$ for the different galaxy populations confirm slopes within $1 \sigma$ of zero (all galaxies: $-2.36 \times 10^{-5} \pm 1.84 \times 10^{-4}$ ), i.e., the observed trend between (F390W-F814W) color and $\mathrm{R}_{\text {proj }}$ is driven by stellar mass.

We test whether $M_{\star}$ can also explain the decreasing fraction of members with pockets of UV emission (clumpy) with decreasing group distance (Fig. 4 . 3.1). The clumpy members tend to have lower stellar mass compared to the smooth members with average $\log \left(\mathrm{M}_{\star} / \mathrm{M}_{\odot}\right)$ of $9.9 \pm 0.48$ and $10.7 \pm 0.51$, respectively. Least-squares fits to $\delta_{\text {col }}\left(M_{\star}\right)$ and $\mathrm{R}_{\text {proj }}$ of both clumpy and smooth members confirm trends consistent with zero, (clumpy: $4.39 \pm 4.48 \times 10^{-4}$, smooth: $-2.33 \pm 2.00 \times 10^{-4}$ ), i.e. the correlation between UV morphology and projected group distance disappears once we account for stellar mass.

To confirm that stellar mass is the key parameter driving this relation, we test whether projected groupcentric distance provides an equally good explanation of the observed correlation. The correlation between color and radius is much weaker than the trend with mass. If we define $\delta_{\text {col }}\left(R_{\text {proj }}\right)$ as the difference between the galaxy's measured (F390W-F814W) color and the expected color given its $R_{\text {proj }}$ from Eq. 1, then the residual scatter is appreciably larger (0.74 versus 0.52$)$ and $\delta_{\text {col }}\left(R_{\text {proj }}\right)$ is strongly correlated with stellar mass (slope of $0.83 \pm 0.08)$.

We find that the primary predictor for a galaxy's total (F390W-F814W) color and UV morphology is its stellar mass, i.e. mass-quenching (Peng et al. 2010). The increasing fraction of massive galaxies in the core is due to this environmental effect, whereas the increasing fraction of passive galaxies merely reflects mass. Our results in the group environment of SG1120 mirror results by Muzzin et al. (2012) for galaxy clusters at $z \sim 1$.

\subsection{Internal Color Gradients}

With high-resolution imaging from HST, we map internal color gradients at multiple half-light radii for individual supergroup galaxies. We measure the (F390WF814W) at three circular annuli corresponding to 1.0, 1.75 , and 2.5 half-light radii, where $\mathrm{r}_{1 / 2}$ is measured in the F814W imaging. While other studies use more annuli to measure internal color gradients (e.g. Tamura et al. 2000; Welikala \& Kneib 2012, Allen et al. 2015), we focus our analysis on three radii to ensure a robust comparison of the galaxies' disks to their centers, while ensuring that measurement apertures are larger than the PSF for both filters.

We stack internal color gradients for the clumpy and smooth supergroup galaxies (Fig. 9) and confirm that smooth members are $\sim 1$ dex redder than clumpy mem-

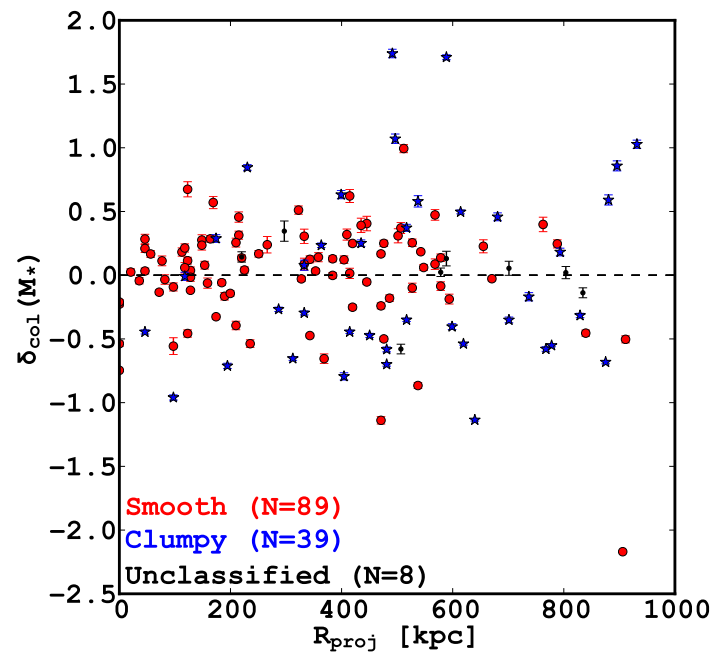

FIG. 8.- Color deviation $\delta_{\text {col }}\left(M_{\star}\right)$ is the difference between a galaxy's measured (F390W-F814W) color and the expected value based on its stellar mass (Eq. 2). Correcting for the stellar mass dependence removes any trend betwen $\delta_{\mathrm{col}}\left(M_{\star}\right)$ and $\mathrm{R}_{\text {proj }}$; this also holds for the clumpy (stars) and smooth (large circles) members. Least-squares fits to $\delta_{\text {col }}\left(M_{\star}\right)-\mathrm{R}_{\text {proj }}$ for the different galaxy populations confirm slopes within $1 \sigma$ of zero (slope of $-2.36 \times 10^{-5} \pm 1.84 \times 10^{-4}$ ). Thus the correlation between total galaxy color and stellar mass explains the correlation between total galaxy color and projected group distance (Fig. 6).

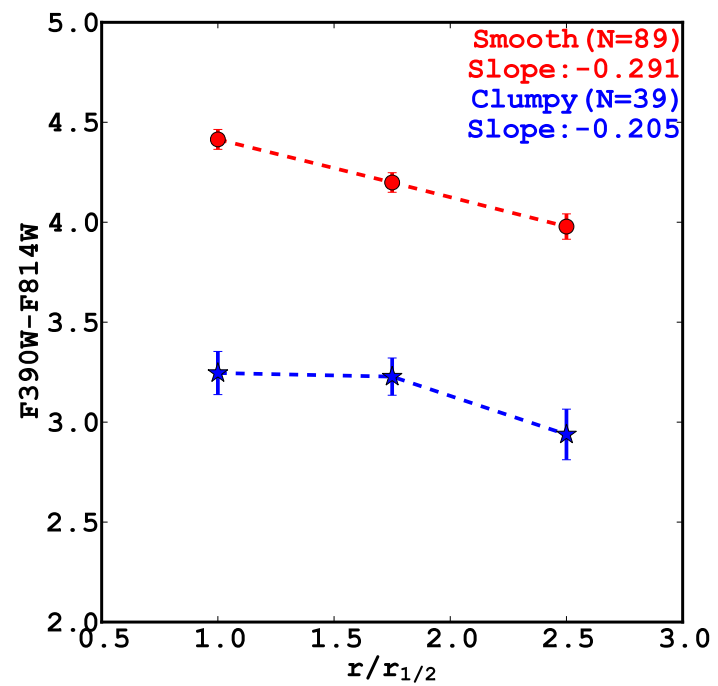

FIG. 9.- With HST's high spatial resolution, we are able to measure internal color gradients and show the stacks for clumpy (stars) and smooth (circles) supergroup members. The smooth members are $\sim 1$ dex redder, and both smooth and clumpy members have shallow gradients.

bers. The average (F390W-F814W) color in each annulus is measured from the galaxy distribution (either clumpy or smooth) and the corresponding error is determined from 1000 boot-strapped realizations. The gradients of clumpy and smooth galaxies have slopes of $-0.205 \pm 0.104$ and $-0.281 \pm 0.002$, respectively.

We also stack and measure internal color gradients for the $24 \mu \mathrm{m}$-detected and non-IR members. As expected, the $24 \mu \mathrm{m}$ members are bluer (F390W-F814W) throughout their galaxy disks and centers compared to the nonIR members. 


\section{DISCUSSION}

A number of physical mechanisms continue to be explored to explain the changes in galaxy properties with environment. Ram pressure stripping (Gunn \& Gott III 1972), tidal interactions with other galaxies or with the cluster potential (Moore et al. 1998), morphological quenching (Martig et al. 2009), and environmental strangulation (Larson et al. 1980) each provide different processes that can explain the observed differences in UV emission (see Boselli \& Gavazzi 2006 for a comprehensive review of such mechanisms). However, given the breadth of these studies and the range of (sometimes) contradictory conclusions, we do not attempt to solve this issue definitively here with only one system.

Instead, we consider a few clues from supergroup SG1120. The galaxies are spatially segregated by UV emission even before the cluster forms (Figs. 2 \& 6), and stellar mass is the key. Most of the members with pockets of UV-bright star formation ("clumpy") tend to be fainter (lower-mass) galaxies (Fig. 5), and a statistically significantly trend between $(\mathrm{F} 390 \mathrm{~W}-\mathrm{F} 814 \mathrm{~W})$ color and stellar mass (Fig. 7. Eq. 2) drives this relation. Both results reinforce the link between increasing stellar mass and decreasing star formation ("mass-quenching"; Peng et al. 2010).

However, this is not to say that environment is unimportant (see also Cooper et al.|2010). First, there is masssegregation in the group environment where the fraction of massive galaxies increases with decreasing $R_{\text {proj }}$. Second, we find for the first time group galaxies with $\mathrm{UV}$ tails and asymmetric UV emission (Fig. 2, top 3 rows) similar to those of jellyfish galaxies. These jellyfish candidates are interpreted as signs of shocked gas fronts in merging clusters (Owers et al. 2012) which is consistent with the incipient merger of the four X-ray luminous galaxy groups that make up SG1120. Third, the slope of the UV color-stellar mass relation in SG1120 may be different from that of the field.

The increasing fraction of UV emitting/clumpy members with increasing projected group distance (Figs. 4 \& 6) mirrors the same trend for $24 \mu \mathrm{m}$ members (Tran et a. 2009). The shallow internal color gradients (Fig. 9) of both the clumpy and smooth populations suggest that quenching should happen uniformly throughout a galaxy's disk and core once it is within $\sim 2 \times R_{200}$ of the group core (Fig. 4). The UV emitting galaxies tend to have lower stellar masses and can thus fade into the faint quiescent members that are prevalent in lowredshift clusters (De Lucia \& Blaizot 2007). Alternatively, these quenched galaxies can merge to form more massive (quiescent) members in the group cores (Tran et al. 2008).

\section{CONCLUSIONS}

We map the UV emission in a supergroup at $z=0.37$ by combining high-resolution imaging from the Hubble Space Telescope with extensive ground-based multi-band imaging and spectroscopy. SG1120 is composed of four
X-ray luminous galaxy groups that will merge and form a galaxy cluster by $z \sim 0$ (Gonzalez et al. 2005). We use WFC3/F390W and ACS/F814W imaging of supergroup galaxies to measure total (F390W-F814W) colors, visually separate UV morphology into "clumpy" and "smooth" systems, and measure internal color gradients.

These observations indicate that stellar mass is the primary predictor of UV emission in the supergroup galaxies ("mass-quenching") while environment drives the higher fraction of massive galaxies in the group cores.

We show for the first time that several group members have UV morphologies similar to the jellyfish galaxies in massive X-ray luminous clusters. The incipient merger of the four groups in SG1120 is consistent with the interpretation that jellyfish galaxies are signs of gas shocks due to mergers.

Approximately $30 \%(38 / 128)$ of the visually classified supergroup galaxies have pockets of UV emission ("clumpy"; Fig. 2), a fraction that is comparable to the $24 \mu \mathrm{m}$ members (32\%; Tran et al. 2009). The clumpy UV members have shallow internal (F390W-F814W) color gradients (Fig. 9).

To measure UV properties as a function of projected group distance $\left(\mathrm{R}_{\text {proj }}\right)$, we stack the four galaxy groups into a single system. We find that the UV emission, as measured by the total (F390W-F814W) color and by the clumpy/smooth classification, is strongly correlated with projected group distances (Figs. $4 \& 6$ ). We show that both of these trends in $\mathrm{UV}$ with $\mathrm{R}_{\text {proj }}$ are driven by the strong underlying correlation between (F390W-F814W) and stellar mass: group galaxies with UV emission tend to be lower luminosity/lower-mass members, and these systems are more common at $R_{\text {proj }}>R_{200}$ (Figs. $7 \& 8$ ).

However, we do find evidence of environmental processes. Most importantly, the higher density environment of the group cores $\left(\mathrm{R}_{\text {proj }}<R_{200}\right)$ means an increase in the fraction of members that are massive and red/smooth (mass-segregation). Also, the supergroup contains jellyfish candidates whose UV morphologies likely are shaped by interactions with the intragroup gas.

Our analysis of the supergroup galaxies indicate that stellar mass is the primary predictor of a galaxy's UV properties (mass-quenching), but it is environment that regulates the galaxy mass distribution and shapes jellyfish galaxies.

We are grateful to J. Moustakas and D. Zaritsky for their contributions to the SG1120 data analysis and catalogs. We thank L. Alcorn, B. Forrest, and Jimmy for helpful comments on the data analysis and manuscript. J. M. and K. T. acknowledge support for Program number HST-GO-12470 provided by NASA through a grant from the Space Telescope Science Institute, which is operated by the Association of Universities for Research in Astronomy, Incorporated, under NASA contract NAS526555.

Facilities: VLT (VIMOS), VLT (FORS2), Magellan (LDSS3), KPNO (Mayall 4m), HST (ACS, WFC3), SST (MIPS), CXO (ACIS), Keck (LRIS).

\section{REFERENCES}

Abadi, M. G., Moore, B., \& Bower, R. G. 1999, Monthly Notices of the Royal Astronomical Society, 308, 947
Allen, R. J., Kacprzak, G. G., Spitler, L. R., et al. 2015, ApJ, 1 
Balogh, M. L., Baldry, I. K., Nichol, R., et al. 2004, The Astrophysical Journal, 615, L101

Bekki, K., Couch, W. J., \& Shioya, Y. 2002, ApJ, 577, 651

Bell, E., Wolf, C., Meisenheimer, K., et al. 2004, ApJ, 608, 752

Bertin, E., \& Arnouts, S. 1996, Astronomy and Astrophysics Supplement Series, 117, 393

Boselli, A., \& Gavazzi, G. 2006, Publications of the Astronomical Society of the Pacific, 118, 517

Butcher, H., \& Oemler, A., J. 1978, The Astrophysical Journal, 219, 18

Byrd, G., \& Valtonen, M. 1990, The Astrophysical Journal, 350, 89

Cooper, M. C., Coil, A. L., Gerke, B. F., et al. 2010, Monthly Notices of the Royal Astronomical Society, 409, 337

De Lucia, G., \& Blaizot, J. 2007, Monthly Notices of the Royal Astronomical Society, 375, 2

Dressler, A. 1980, The Astrophysical Journal, 236, 351

Dressler, A., Oemler, A., Jr., et al. 1997, Astrophysical Journal, 490, 577

Ebeling, H., Stephenson, L. N., \& Edge, A. C. 2014, The Astrophysical Journal, 781, L40

Freeland, E., Tran, K.-V. H., Irwin, T., et al. 2011, The Astrophysical Journal, 742, L34

Gallazzi, A., Bell, E. F., Wolf, C., et al. 2009, The Astrophysical Journal, 690, 1883

Gonzalez, A., Tran, K.-V., Conbere, M., Zaritsky, D., \& Journal, T. A. 2005, ApJL, 624, L73

Gonzalez, A. H., Zaritsky, D., Dalcanton, J., \& Nelson, A. 2001, ApJS, 137, 117

Gunn, J., \& Gott III, J. 1972, ApJ, 176, 1

Hashimoto, Y., Oemler, Jr., A., Lin, H., \& Tucker, D. L. 1998, The Astrophysical Journal, 499, 589

Iovino, A., Cucciati, O., Scodeggio, M., et al. 2010, Astronomy and Astrophysics, 509, A40

Just, D. W., Zaritsky, D., Tran, K.-V. H., et al. 2011, The Astrophysical Journal, 740, 54

Kautsch, S. J., Gonzalez, A. H., Soto, C. a., et al. 2008, The Astrophysical Journal Letters, 688, L5

Kennicutt Jr, R. C., Evans II, N. J., Kennicutt, R. C., \& Evans, N. J. 2012, Annual Review of Astronomy and Astrophysics, 50 531

Kodama, T., Smail, I., Nakata, F., Okamura, S., \& Bower, R. G. 2001, The Astrophysical Journal, 562, L9

Koyama, Y., Kodama, T., Shimasaku, K., et al. 2010, Monthly Notices of the Royal Astronomical Society, 403, 1611

Larson, R. B., Tinsley, B. M., \& Caldwell, C. N. 1980, The Astrophysical Journal, 237, 692

Martig, M., Bournaud, F., Teyssier, R., \& Dekel, A. 2009, The Astrophysical Journal, 707, 250
Meurer, G. R., Heckman, T. M., \& Calzetti, D. 1999, The Astrophysical Journal, 521, 64

Moore, B., Lake, G., \& Katz, N. 1998, The Astrophysical Journal, 495,139

Muzzin, A., Wilson, G., Yee, H. K. C., et al. 2012, The Astrophysical Journal, 746, 188

Owers, M. S., Couch, W. J., Nulsen, P. E. J., \& Randall, S. W. 2012, The Astrophysical Journal, 750, L23

Peebles, P. J. E. 1970, The Astronomical Journal, 75, 13

Peng, Y.-j., Lilly, S. J., Kovač, K., et al. 2010, The Astrophysical Journal, 721, 193

Postman, M., Franx, M., Cross, N. J. G., et al. 2005, The

Astronomical Journal, 623, 721

S. Gonzaga, W. Hack, A. Fruchter, J. M. 2012, STScI, 63

Saintonge, A., Tran, K.-V. H., \& Holden, B. P. 2008, ArXiv eprints, 685,5

Skelton, R. E., Whitaker, K. E., Momcheva, I. G., et al. 2014,

The Astrophysical Journal Supplement Series, 214, 24

Smit, M., Schrabback, T., Velander, M., et al. 2015, Astronomy \& Astrophysics, 1

Smith, R. J., Lucey, J. R., Price, J., Hudson, M. J., \& Phillipps, S. 2012, Monthly Notices of the Royal Astronomical Society, 419,3167

Smith, R. J., Lucey, J. R., Hammer, D., et al. 2010, Monthly Notices of the Royal Astronomical Society, 408, 1417

Stanford, S., Eisenhardt, P. R., \& Dickinson, M. 1998, ApJ, 10

Tamura, N., Kobayashi, C., Arimoto, N., Kodama, T., \& Ohta, K. 2000, The Astronomical Journal, 119, 2134

Tonnesen, S., Bryan, G. L., \& van Gorkom, J. H. 2007, The Astrophysical Journal, 671, 1434

Tran, K.-V., Saintonge, A., Moustakas, J., et al. 2009, ApJ, 705, 809

Tran, K.-V. H., Moustakas, J., Gonzalez, A. H., et al. 2008, ApJ, 17

Tran, K.-V. H., Simard, L., Zabludoff, A. I., \& Mulchaey, J. S. 2001, ApJ, 549, 172

van Dokkum, P. G., Franx, M., Kelson, D. D., et al. 1998, ApJ, 500,714

Welikala, N., \& Kneib, J.-p. 2012, arXiv:1202.0494, 13

Wilman, D. J., Pierini, D., Tyler, K., et al. 2008, The Astrophysical Journal, 680, 1009

Wuyts, S., Förster Schreiber, N. M., Genzel, R., et al. 2012, The Astrophysical Journal, 753, 114

Zabludoff, A. I., \& Mulchaey, J. S. 1998, ApJ, 496, 39 
TABLE 2

Observed Properties of Supergroup Members

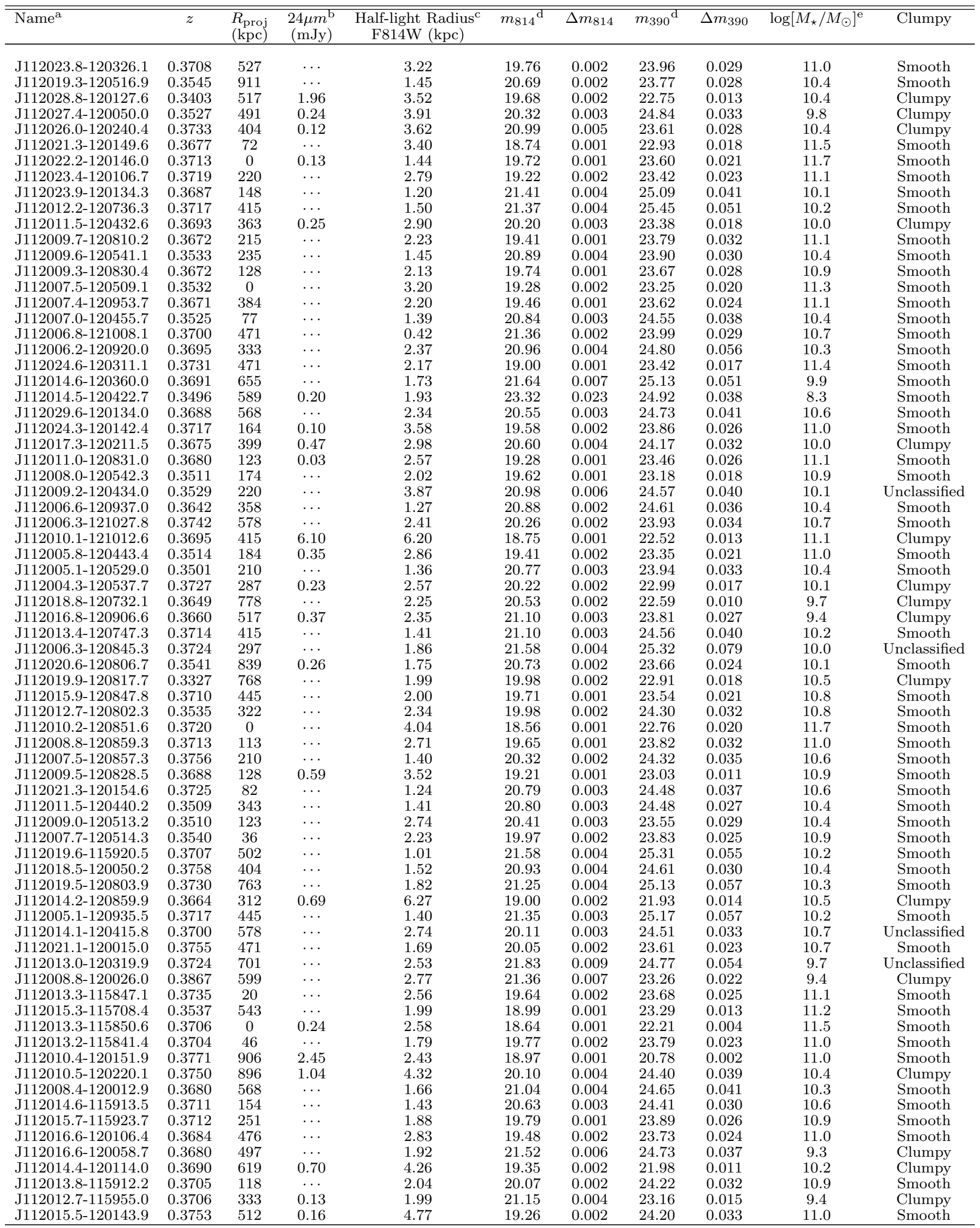


TABLE 2

Observed Properties of Supergroup Members

\begin{tabular}{|c|c|c|c|c|c|c|c|c|c|c|}
\hline J112017.8-120024.8 & 0.3744 & 537 & $\ldots$ & 3.02 & 21.13 & 0.006 & 23.21 & 0.022 & 9.4 & Smooth \\
\hline J112017.9-115942.3 & 0.3690 & 435 & $\cdots$ & 1.03 & 21.70 & 0.005 & 25.47 & 0.056 & 10.1 & Smooth \\
\hline J112008.9-120931.1 & 0.3721 & 225 & $\ldots$ & 2.45 & 20.04 & 0.002 & 23.89 & 0.024 & 10.8 & Smooth \\
\hline $\mathrm{J} 112008.9-120819.7$ & 0.3683 & 189 & $\cdots$ & 1.62 & 20.34 & 0.002 & 23.83 & 0.029 & 10.5 & Smooth \\
\hline J112009.4-120906.4 & 0.3673 & 97 & $\ldots$ & 2.62 & 20.09 & 0.001 & 23.76 & 0.032 & 10.7 & Smooth \\
\hline J112009.7-120920.0 & 0.3652 & 148 & $\ldots$ & 1.51 & 20.40 & 0.002 & 24.35 & 0.038 & 10.6 & Smooth \\
\hline J112010.7-121104.0 & 0.3500 & 681 & 0.53 & 4.63 & 19.86 & 0.002 & 23.98 & 0.031 & 10.6 & Clumpy \\
\hline J112010.0-120601.5 & 0.3580 & 333 & $\ldots$ & 1.72 & 21.86 & 0.005 & 24.62 & 0.040 & 9.7 & Clumpy \\
\hline J112011.0-121108.5 & 0.3510 & 701 & $\cdots$ & 2.59 & 20.63 & 0.003 & 22.74 & 0.022 & 9.5 & Clumpy \\
\hline J112010.9-120836.3 & 0.3680 & 97 & $\ldots$ & 0.86 & 22.14 & 0.005 & 24.88 & 0.066 & 10.0 & Smooth \\
\hline J112011.5-120928.7 & 0.3692 & 215 & $\ldots$ & 2.65 & 20.94 & 0.003 & 24.91 & 0.040 & 10.3 & Smooth \\
\hline J112011.5-120828.3 & 0.3702 & 159 & $\ldots$ & 1.90 & 20.99 & 0.003 & 24.28 & 0.040 & 10.1 & Smooth \\
\hline J112011.3-121114.2 & 0.3667 & 737 & $\ldots$ & 2.42 & 22.06 & 0.009 & 23.80 & 0.031 & 9.0 & Clumpy \\
\hline J112015.1-120722.9 & 0.3720 & 589 & 0.17 & 3.43 & 20.90 & 0.004 & 24.85 & 0.057 & 10.3 & Unclassified \\
\hline $\mathrm{J} 112014.8-120742.3$ & 0.3692 & 507 & $\ldots$ & 2.35 & 20.75 & 0.003 & 24.07 & 0.038 & 10.4 & Unclassified \\
\hline J112015.1-120746.3 & 0.3720 & 507 & $\ldots$ & 2.24 & 20.53 & 0.003 & 24.60 & 0.043 & 10.6 & Smooth \\
\hline J112015.9-120712.7 & 0.3706 & 671 & $\ldots$ & 2.23 & 19.07 & 0.001 & 23.13 & 0.016 & 11.2 & Smooth \\
\hline J112016.5-120812.8 & 0.3713 & 527 & $\ldots$ & 2.30 & 20.63 & 0.003 & 24.09 & 0.037 & 10.4 & Smooth \\
\hline J112017.1-120940.2 & 0.3512 & 589 & 0.46 & 4.90 & 19.67 & 0.002 & 24.26 & 0.019 & 9.9 & Clumpy \\
\hline J112017.9-120554.8 & 0.3900 & 834 & $\ldots$ & 3.15 & 22.19 & 0.012 & 23.89 & 0.037 & 9.0 & Unclassified \\
\hline J112019.5-120536.0 & 0.3746 & 932 & 1.27 & 4.98 & 19.22 & 0.001 & 23.88 & 0.032 & 10.6 & Clumpy \\
\hline J112020.0-120805.8 & 0.3449 & 793 & $\ldots$ & 2.27 & 22.14 & 0.008 & 24.18 & 0.029 & 9.0 & Clumpy \\
\hline J112010.9-120234.9 & 0.3744 & 829 & 0.61 & 5.16 & 20.10 & 0.004 & 22.67 & 0.017 & 9.9 & Clumpy \\
\hline J112009.6-120114.1 & 0.3698 & 788 & $\ldots$ & 2.14 & 20.16 & 0.003 & 24.17 & 0.032 & 10.7 & Smooth \\
\hline J112010.9-115752.1 & 0.3692 & 353 & $\ldots$ & 3.06 & 19.02 & 0.001 & 23.28 & 0.019 & 11.4 & Smooth \\
\hline J112023.3-120329.7 & 0.3758 & 537 & $\ldots$ & 2.89 & 21.14 & 0.005 & 24.47 & 0.044 & 9.8 & Clumpy \\
\hline J112010.5-120223.8 & 0.3744 & 880 & $\ldots$ & 3.07 & 21.59 & 0.011 & 24.33 & 0.038 & 9.3 & Clumpy \\
\hline J112010.9-120207.8 & 0.3770 & 875 & $\ldots$ & 3.98 & 20.73 & 0.005 & 22.61 & 0.012 & 9.6 & Clumpy \\
\hline J112010.9-120315.3 & 0.3497 & 640 & 0.21 & 2.68 & 20.32 & 0.003 & 21.81 & 0.009 & 9.7 & Clumpyf \\
\hline J112011.5-115935.2 & 0.3681 & 266 & $\ldots$ & 0.84 & 21.95 & 0.006 & 25.50 & 0.064 & 10.0 & Smooth \\
\hline J112012.4-120250.3 & 0.3747 & 804 & 0.17 & 3.43 & 20.51 & 0.004 & 24.65 & 0.048 & 10.5 & Unclassified \\
\hline J112012.6-115910.6 & 0.3712 & 118 & $\ldots$ & 1.89 & 20.24 & 0.002 & 23.97 & 0.032 & 10.7 & Clumpy \\
\hline J112013.2-120345.2 & 0.3684 & 614 & 0.62 & 6.39 & 19.47 & 0.003 & 23.04 & 0.015 & 10.1 & Clumpy ${ }^{f}$ \\
\hline J112014.0-115744.5 & 0.3339 & 343 & 0.07 & 2.82 & 19.93 & 0.003 & 23.20 & 0.016 & 10.7 & Smooth \\
\hline J112014.0-115913.1 & 0.3692 & 128 & $\ldots$ & 2.02 & 20.17 & 0.002 & 24.02 & 0.032 & 10.8 & Smooth \\
\hline J112014.1-115814.3 & 0.3325 & 195 & $\ldots$ & 3.72 & 21.14 & 0.007 & 22.70 & 0.019 & 9.4 & Clumpy \\
\hline J112014.5-115808.9 & 0.3742 & 230 & 0.19 & 3.47 & 20.11 & 0.003 & 23.77 & 0.022 & 9.9 & Clumpy ${ }^{f}$ \\
\hline J112014.3-115739.8 & 0.3713 & 369 & $\ldots$ & 3.76 & 21.60 & 0.011 & 23.83 & 0.036 & 9.4 & Smooth \\
\hline J112014.7-120117.2 & 0.3701 & 594 & $\ldots$ & 1.77 & 20.89 & 0.004 & 24.21 & 0.038 & 10.3 & Smooth \\
\hline J112014.6-115718.1 & 0.3741 & 481 & 0.27 & 4.27 & 20.05 & 0.003 & 22.19 & 0.013 & 9.9 & Clumpy \\
\hline J112014.9-115825.6 & 0.3736 & 174 & 0.12 & 3.14 & 21.00 & 0.004 & 23.89 & 0.030 & 9.7 & Clumpy \\
\hline J112015.9-115855.1 & 0.3692 & 200 & $\ldots$ & 1.79 & 19.73 & 0.001 & 23.49 & 0.021 & 10.9 & Smooth \\
\hline J112016.8-120156.3 & 0.3712 & 420 & $\ldots$ & 2.41 & 19.44 & 0.002 & 23.18 & 0.019 & 11.0 & Smooth \\
\hline J112017.3-120132.4 & 0.3711 & 384 & $\ldots$ & 1.81 & 19.65 & 0.002 & 23.63 & 0.022 & 11.0 & Smooth \\
\hline J112017.6-120241.1 & 0.3338 & 450 & 1.04 & 4.53 & 20.29 & 0.003 & 22.42 & 0.016 & 9.7 & Clumpy \\
\hline $\mathrm{J} 112017.7-115757.2$ & 0.3549 & 435 & 0.39 & 2.86 & 20.39 & 0.003 & 23.24 & 0.026 & 9.7 & Clumpy \\
\hline J112018.2-120256.8 & 0.3761 & 476 & 0.10 & 6.56 & 20.23 & 0.005 & 23.01 & 0.019 & 9.9 & Smooth \\
\hline J112017.8-120230.5 & 0.3708 & 410 & $\ldots$ & 1.55 & 20.84 & 0.004 & 24.82 & 0.043 & 10.5 & Smooth \\
\hline J112018.5-120324.3 & 0.3737 & 578 & $\ldots$ & 4.41 & 19.91 & 0.003 & 23.92 & 0.030 & 10.8 & Smooth \\
\hline J112019.2-120230.7 & 0.3701 & 328 & $\ldots$ & 1.89 & 19.73 & 0.002 & 23.62 & 0.020 & 10.9 & Smooth \\
\hline J112020.1-120017.2 & 0.3692 & 481 & 0.19 & 3.70 & 20.12 & 0.003 & 22.43 & 0.019 & 9.9 & Clumpy \\
\hline J112021.6-120143.2 & 0.3716 & 46 & $\ldots$ & 1.37 & 20.66 & 0.003 & 24.68 & 0.031 & 10.7 & Smooth \\
\hline J112021.1-120135.5 & 0.3677 & 97 & $\ldots$ & 2.84 & 21.42 & 0.007 & 22.74 & 0.019 & 9.4 & Clumpy ${ }^{\mathrm{f}}$ \\
\hline J112021.3-120215.6 & 0.3735 & 169 & $\ldots$ & 2.48 & 20.43 & 0.003 & 24.76 & 0.048 & 10.7 & Smooth \\
\hline $\mathrm{J} 112021.8-120208.2$ & 0.3712 & 118 & $\ldots$ & 1.67 & 20.86 & 0.004 & 24.57 & 0.041 & 10.5 & Smooth \\
\hline $\mathrm{J} 112022.7-120307.5$ & 0.3742 & 420 & $\ldots$ & 3.82 & 18.66 & 0.001 & 23.19 & 0.020 & 11.5 & Smooth \\
\hline $\mathrm{J} 112021.7-120250.4$ & 0.3686 & 333 & $\ldots$ & 1.64 & 21.02 & 0.003 & 24.70 & 0.046 & 10.4 & Smooth \\
\hline J112022.6-120153.2 & 0.3455 & 46 & 0.90 & 2.45 & 20.21 & 0.002 & 22.61 & 0.016 & 9.9 & Clumpy $\mathrm{f}$ \\
\hline J112022.9-120151.4 & 0.3714 & 56 & $\ldots$ & 0.99 & 20.30 & 0.002 & 24.29 & 0.028 & 10.8 & Smooth \\
\hline J112022.7-120141.4 & 0.3708 & 46 & $\ldots$ & 1.10 & 21.04 & 0.003 & 24.94 & 0.037 & 10.4 & Smooth \\
\hline J112023.2-120127.6 & 0.3731 & 123 & $\ldots$ & 1.26 & 21.29 & 0.004 & 25.38 & 0.059 & 10.2 & Smooth \\
\hline J112025.0-120324.1 & 0.3723 & 548 & $\ldots$ & 2.35 & 19.39 & 0.001 & 23.53 & 0.022 & 11.2 & Smooth \\
\hline J112024.2-120316.1 & 0.3703 & 486 & $\ldots$ & 1.61 & 20.00 & 0.001 & 23.71 & 0.026 & 10.9 & Smooth \\
\hline
\end{tabular}

a Names follow the SDSS format: JHHMMSS.s \pm DDMMSS.s for R.A. and decl. in sexagesimal units.

b Errors corresponding to adopting different conversion factors for $\mathrm{F}_{24 \mu m}$ to $\mathrm{F}_{8-1000 \mu m}$ are $\sim 10-20 \%$.

${ }^{c}$ For galaxies with $r_{1 / 2}>1 \mathrm{kpc}$, Source Extractor measurements of the error on their spheroid radii are $\sim 1-8 \%$.

d Magnitudes are observed-frame.

e See Tran et al. (2009) for errors in mass calculation.

f Jellyfish candidate 